\section{Supramolecular hydrogels based on antimycobacterial amphiphiles $\dagger$}

\author{
Andreas Bernet, ${ }^{*}$ Marina Behr and Hans-Werner Schmidt* \\ Received 23rd December 2011, Accepted 15th March 2012 \\ DOI: $10.1039 / \mathrm{c} 2 \mathrm{sm} 07456 \mathrm{~g}$
}

\begin{abstract}
Synthetic modification of antimycobacterial 4-alkoxy-anilines yields low molecular weight amphiphiles. The bioactivity is retained in these amphiphiles and shows alkyl chain length specificity. The self-assembly behaviour in aqueous media was investigated. It was found that several of these new compounds are capable of forming thermoreversible supramolecular hydrogels in alkaline aqueous media. The initial drug can be released from the hydrogel by thermal hydrolysis.
\end{abstract}

Several severe diseases are caused by mycobacteria. The most important pathogenic mycobacteria species are $M$. leprae (leprosy), $M$. bovis (bovine tuberculosis) and M. tuberculosis (tuberculosis). Especially tuberculosis is regarded as one of the most threatening diseases of the $21^{\text {st }}$ century because this lung disease causes more than 9000000 new infections and 2000000 deaths per year. ${ }^{1}$ Treatment of tuberculosis is challenging, as the high chemical stability of the cell wall of the germs makes it difficult to find effective antimycobacterial agents. Furthermore, long-term treatment is necessary, and the germs are known to develop multi-resistance against antimycobacterial drugs. Therefore there is an urgent need for new antimycobacterial drugs and for the modifications of known antimycobacterial substances.

A common approach for advanced drug delivery applications is the synthetic modification of known bioactive drugs, thus transferring them into "cargo"-molecules. Compared to the underlying starting molecule, the resulting drug derivatives often benefit in terms of bio-applications as improved properties like bioavailability or lower toxicity can be achieved. Under appropriate conditions, the drug can be released from the cargo molecule via external triggers like thermal hydrolysis or enzymatic cleavage. A well-known example is acetylated salicylic acid (aspirin) that is derived from the natural product salicylic acid present in willow bark in the form of its $\beta$-glycoside, salicin.

Especially in the application of supramolecular hydrogels as drug delivery systems, encouraging advances have been made within the last decade. Supramolecular hydrogels (SHGs) or "molecular

Macromolecular Chemistry I, Bayreuther Institut für Makromolekülforschung (BIMF), Bayreuther Zentrum für Kolloide und Grenzflächen (BZKG), University of Bayreuth, D-95440 Bayreuth, Germany. E-mail: andreas.bernet@uni-bayreuth.de; hans-werner.schmidt@uni-bayreuth.de; Fax: +49 (0)921 553206; Tel: +49(0)921553200

$\dagger$ Electronic supplementary information (ESI) available: All experimental details. See DOI: 10.1039/c2sm07456g hydrogels" are based on low molecular weight compounds that are capable of directed self-assembly in aqueous media due to noncovalent and therefore generally reversible intermolecular interactions. The resulting three-dimensional network-like aggregates of the hydrogelator molecules entrap the surrounding solvent, and a macroscopic gel sample is formed. ${ }^{2}$ SHGs have been intensively studied, and the first biomedical applications are within reach: the formation of hydrogels can take place under simulated physiological conditions ${ }^{3}$ or in the interior of cells, ${ }^{4}$ and SHGs have been used as scaffolds for cell growth, ${ }^{5,6}$ to reduce scar formation, ${ }^{7}$ for the treatment of wounds $s^{8}$ and as matrices for drug delivery. ${ }^{9-13}$ Furthermore, a hydrogelator bearing a quaternized pyridyl moiety was shown to feature antibacterial activity, ${ }^{14}$ and Fmoc-peptide based hydrogelators with anti-inflammatory properties were reported. ${ }^{15}$ However, hydrogelators derived from small drug molecules are very rare. To the best of our knowledge, only four SHGs based on the drug molecules ibuprofen, ${ }^{16}$ taxol, ${ }^{17}$ acetaminophen (paracetamol) ${ }^{18}$ and vancomycin ${ }^{19}$ have been reported to date.

In this paper, we investigate a class of amphiphiles derived from 4alkoxyanilines. These aniline derivatives have been reported to show antimycobacterial activity against Mycobacterium smegmatis in dependence of the alkyl chain length with the octyloxy derivative featuring the maximal inhibition of growth. ${ }^{20}$

We decided to transform these drug molecules into diglycolic acid monoamide derivatives (Fig. 1) for the following reasons: (a) our previous work has shown that dicarboxylic acid monoamide amphiphiles are promising candidates for supramolecular hydrogel formation; (b) amidation of the aromatic amine moiety should reduce the issue of oxidative degradation; (c) the bio-related diglycolic monoamide structure is likely to be accessible to enzymatic cleavage and (d) changing the initial alkaline drug molecules into acidic amphiphiles should result in a change of the in vivo uptake behavior.

Synthesis of the diglycolic acid monoamides 1-6 has been successfully accomplished in a straightforward way based on commercially available starting materials. Reaction of diglycolic acid anhydride with 4-alkoxyanilines in dichloromethane at room temperature yields the corresponding diglycolic acid monoamides. Due to the formation of an aromatic amide bond, the products tend to aggregate and can be easily isolated in high purity and good yields ( $82 \%$ average) by precipitation with cyclohexane. Size exclusion chromatographic analysis (SEC) confirmed the absence of any impurities or byproducts.

Evaluation of the bioactivity of the synthesized amphiphiles 1-6 was carried out by an antimicrobial broadband screening via agar 

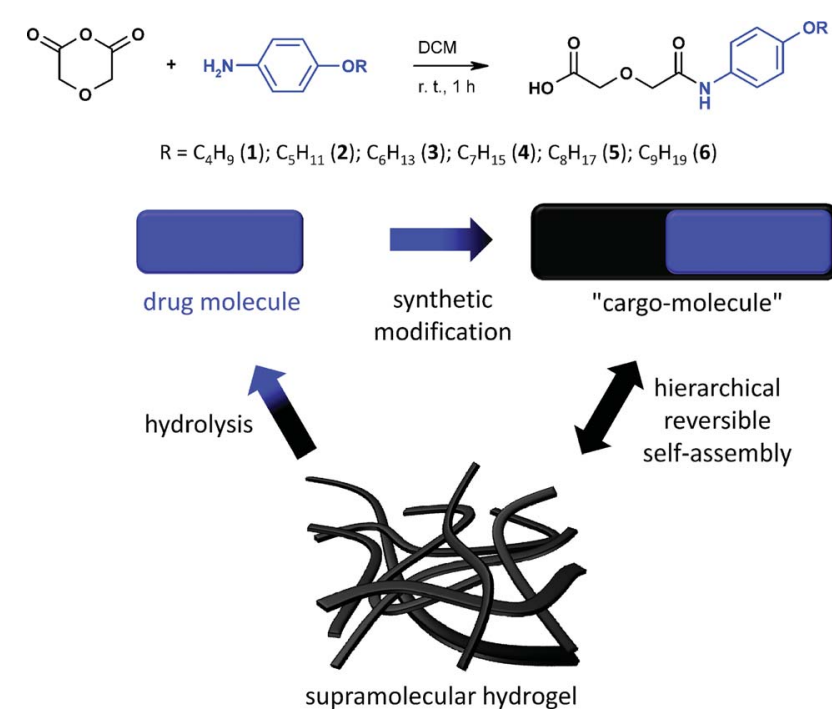

Fig. 1 Synthesis of the bioactive alkoxyaniline-containing low molecular weight amphiphiles 1-6 (top) and schematic representation of the self-assembly/drug release process of the "cargo-molecules" (bottom).

diffusion assay with different strains of bacteria and fungi (see ESI, Table S1 $\dagger$ ). Compounds $\mathbf{5}$ and $\mathbf{6}$ show antibacterial effects against Bacillus subtilis, Staphylococcus aureus, vancomycin-resistant Enterococcus faecalis (VRE) and multidrug-resistant Staphylococcus aureus (MRSA). Candida albicans is affected by compounds $\mathbf{3 - 6}$, whereas all compounds except $\mathbf{6}$ are active against Glomerella. Summarizing, this class of compounds shows specific antimicrobial effects dependent on the alkyl chain length.

Assays for antiproliferative and cytotoxic properties of the compounds 1-6 were carried out by a CellTiter-Blue cell viability assay with HUVEC (human umbilical vein endothelial), K-562 (human immortalized myelogenous leukaemia) and HeLa (human cervix carcinoma) cell lines, respectively. All tested compounds did not show any antiproliferative effect on HUVEC or K-562 cells $\left(\mathrm{GI}_{50}\right.$ values $>50 \mu \mathrm{g} \mathrm{mL}^{-1}(\mathbf{1}-\mathbf{5}), \mathrm{GI}_{50 \text { (HUVEC) }}=48$ (6)). Only compounds 4-6 were moderately to marginally cytotoxic with $\mathrm{CC}_{50}$ values around $40 \mu \mathrm{g} \mathrm{mL}^{-1}$ (see ESI, Table S2 $\dagger$ ).

Antimycobacterial activity of the compounds 1-6 was evaluated via agar diffusion assay against mycobacteria strains $M$. smegmatis, M. aurum, M. vaccae and M. fortuitum (Table 1). All compounds showed antimycobacterial activity against several mycobacteria strains. The octyloxy derivative $\mathbf{5}$ is effective against all four tested strains, and the hexyloxy derivative 3 shows the highest antimycobacterial activity ( $37 \mathrm{~mm}$ zone of growth inhibition in the case of $M$. vaccae). In general, this class of compounds is especially effective against $M$. vaccae and $M$. aurum whereas only compounds 5 and $\mathbf{6}$ showed activity against $M$. smegmatis.

The potential for the formation of supramolecular hydrogels was evaluated by an "inverted test tube" assay. Mixtures of $10 \mathrm{~g} \mathrm{~L}^{-1}$ of amphiphile and pure water or alkaline aqueous solvents $(\mathrm{NaOH}$ at different concentrations from $\mathrm{pH} 11$ to $14, \mathrm{Na}_{2} \mathrm{CO}_{3} \cdot 10 \mathrm{H}_{2} \mathrm{O}(200$ \& $\left.790 \mathrm{~g} \mathrm{~L}^{-1}\right), \mathrm{NaHCO}_{3}\left(100 \mathrm{~g} \mathrm{~L}^{-1}\right), \mathrm{Na}_{3} \mathrm{PO}_{4}\left(260 \mathrm{~g} \mathrm{~L}^{-1}\right)$, and $\mathrm{Na}_{2} \mathrm{HPO}_{4}$ $\left(220 \mathrm{~g} \mathrm{~L}^{-1}\right)$ ) were prepared. After heating to approx. $95^{\circ} \mathrm{C}$ under stirring and subsequently cooling to room temperature, the samples were allowed to stand overnight. A gel state was defined if no gravitational flow was observed upon inversion of the test tube. Three hydrogel systems were successfully identified: 3 in aqueous $\mathrm{NaOH}$ solution ( $\mathrm{pH} 11$ and 12), 5 in aqueous $\mathrm{NaOH}(\mathrm{pH} 11)$ and 6 in aqueous $\mathrm{Na}_{3} \mathrm{PO}_{4}$ solution. Unfortunately, the gel formation behavior of $\mathbf{5}$ and $\mathbf{6}$ seems to be very sensitive to the applied conditions like shearing and temperature profile, and the reproducibility of gel formation tended to be erratic. For this reason we decided to concentrate our investigations on the hydrogel systems based on the hexyloxy-substituted amide $\mathbf{3}$ and aqueous $\mathrm{NaOH}$ solutions.

The influence of both the concentration of $\mathbf{3}$ and the $\mathrm{pH}$ value of the aqueous $\mathrm{NaOH}$ solution on the gel formation has been studied by preparing an array of samples $\left(c(\mathbf{3})=1-10 \mathrm{~g} \mathrm{~L}^{-1}, 1 \mathrm{~g} \mathrm{~L}^{-1}\right.$ increment; $\mathrm{pH}$ range $11-14,0.2$ increment) and investigating the resulting samples after a heating/cooling cycle.

It has to be noted that, due to the initial alkaline conditions, a (partial) deprotonation of the carboxylic groups is reasonable to be assumed. Estimation of the degree of deprotonation (dod) of these samples (see ESI $\dagger$ ) indicates that a certain dod is necessary for the formation of a hydrogel. At $\mathrm{pH} 11.5-11.9(\mathrm{dod}=10-25 \%)$, gels are formed. $\mathrm{pH} 12.0(\mathrm{dod}=31 \%)$ and $12.2(\mathrm{dod}=49 \%)$ result in a mixture of an inhomogeneous gel and sol phase, and at $\mathrm{pH} 12.4$ and above ( $\operatorname{dod} \geq 78 \%$ ), clear fluid solutions are obtained. Although the calculation used is only a very rough estimation, and it is also possible that significant $\mathrm{p} K_{\mathrm{a}}$-shifts occur during the supramolecular aggregation of small $\mathrm{pH}$-sensitive amphiphiles, ${ }^{21}$ these observations clearly indicate that there is a critical dod range for the formation of hydrogels of 3 in aqueous $\mathrm{NaOH}$ solutions.

The gel-sol-transition temperatures $T_{\text {gel }}$ of the identified hydrogel samples of $\mathbf{3}$ were determined by the "falling steel ball" method and are illustrated in Fig. 2. The influence of the concentration of $\mathbf{3}$ on $T_{\text {gel }}$ is marginal, but there is a clear increase in $T_{\text {gel }}$ with increasing $\mathrm{pH}$. The critical gelation concentration (cgc) is 5,6 and $7 \mathrm{~g} \mathrm{~L}^{-1}$ at $\mathrm{pH} 11.5$, 11.7 and 11.9 , respectively.

To get an insight into the $\mathrm{pH}$-sensitive self-assembly behavior of $\mathbf{3}$ in aqueous $\mathrm{NaOH}$ solutions, Fourier transform infrared (FT-IR)

Table 1 Antimycobacterial activity of compounds 1-6 determined by agar diffusion assay ${ }^{a}$

\begin{tabular}{lllll}
\hline Compound & Mycobacterium smegmatis & Mycobacterium aurum & Mycobacterium vaccae & Mycobacterium fortuitum \\
\hline $\mathbf{1}$ & 0 & 0 & $17 \mathrm{P}$ & $15 \mathrm{P}$ \\
$\mathbf{2}$ & 0 & $25(\mathrm{p})$ & $16 \mathrm{P}$ & 0 \\
$\mathbf{3}$ & 0 & $20 \mathrm{p}$ & $20 / 37 \mathrm{p}$ & $15 \mathrm{p}-\mathrm{P}$ \\
$\mathbf{4}$ & 0 & $11 / 17 \mathrm{P}$ & $19 \mathrm{p}$ & $13 \mathrm{p}-\mathrm{P}$ \\
$\mathbf{5}$ & $12 \mathrm{p}$ & $12 / 24 \mathrm{p}$ & $12 \mathrm{p}-\mathrm{P}$ \\
$\mathbf{6}$ & $13 \mathrm{p}$ & $11 / 26 \mathrm{p}$ & $24(\mathrm{p})$ & 0
\end{tabular}

${ }^{a}$ Values: zone of growth inhibition (diameter in $\mathrm{mm}$ ). p: few colonies in the zone of growth inhibition. P: many colonies in the zone of growth inhibition. Solvent: DMSO. $c=1000 \mu \mathrm{g} \mathrm{mL}-1$. 


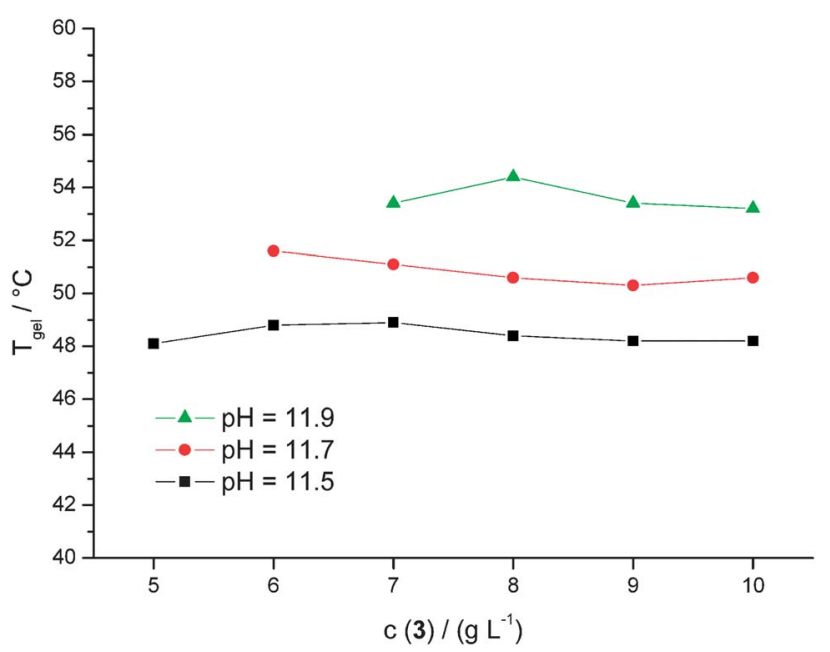

Fig. 2 Influence of concentration of $\mathbf{3}$ and the initial $\mathrm{pH}$ value on the gel-sol transition temperature $T_{\text {gel }}$ of the corresponding hydrogels in aqueous $\mathrm{NaOH}$ solutions, determined by the "falling steel ball" method.

spectroscopy, X-ray diffraction (XRD) and electron microscopy experiments were carried out on samples obtained at different $\mathrm{pH}$ values. In all samples only hydrogen-bonded amide groups are present as proven by corresponding $\nu(\mathrm{N}-\mathrm{H})$ wavenumbers below $3400 \mathrm{~cm}^{-1}$ (see ESI, Fig. S1 $\dagger$ ). FT-IR of 3 as bulk shows a N-Hstretching band $(\nu(\mathrm{N}-\mathrm{H}))$ at $3344 \mathrm{~cm}^{-1}$, whereas in the dried gel sample prepared from $\mathrm{pH} 11.5$ two signals at $3342 \mathrm{~cm}^{-1}$ and $3302 \mathrm{~cm}^{-1}$ are visible. The first peak is not retrievable in the dried gel samples from $\mathrm{pH} 11.7$ and $\mathrm{pH} 11.9$, whereas the second peak is practically unchanged (3301 $\mathrm{cm}^{-1}$ and $3300 \mathrm{~cm}^{-1}$, respectively). This indicates a change in molecular aggregation with change of the $\mathrm{pH}$ value of the aqueous solvent.

$\mathrm{XRD}$ analysis from $\mathbf{3}$ as obtained from synthesis and as wet hydrogel samples in aqueous $\mathrm{NaOH}$ solution at different $\mathrm{pH}$ values $\left(c(\mathbf{3})=10 \mathrm{~g} \mathrm{~L}^{-1}\right)$ were carried out. The diffraction pattern of $\mathbf{3}$ from synthesis clearly indicates a highly ordered crystalline state (see ESI, Fig. S2 $\dagger$ ). The main peak at $4.94^{\circ}(2 \theta)$ corresponds to a Bragg distance $d=1.79 \mathrm{~nm}$ and is in the range of the molecular length of $\mathbf{3}$ $(2.06 \mathrm{~nm})$. In the case of the XRD patterns of the hydrogel samples, the XRD patterns significantly change with the $\mathrm{pH}$ value. The peak at $4.94^{\circ}(2 \theta)$ can also be retrieved in the spectra of the sample prepared from $\mathrm{pH} 11.5$ and 11.7, but vanishes in the case of $\mathrm{pH} 11.9$. In the case of $\mathrm{pH} 11.5,11.7$ and 11.9 a second peak evolves at $2.36^{\circ}$ ( $2 \theta$ ) corresponding to a Bragg distance $d=3.74 \mathrm{~nm}$, which could be attributed to an intercalated or tilted bilayer arrangement of the amphiphilic molecules due to hydrophilic/hydrophobic interactions of polar headgroups and non-polar alkyl chains with the surrounding medium. Increasing the $\mathrm{pH}$ to 12.7 finally results in a homogeneous solution that does not show any recognizable XRD peaks. In combination, the results of the FT-IR and of the XRD studies clearly indicate that the packing mode of the amphiphiles in the aqueous samples is highly dependent on the $\mathrm{pH}$ value during hydrogel preparation and gradually changes from one type to another. We propose that, depending on the $\mathrm{pH}$ value, the hydrogel samples contain a mixture of $\mathbf{3}$ and the corresponding sodium salt $\mathbf{3 N a}$. It is possible that $\mathbf{3 N a}$ acts as a kind of "surfactant" at the surface of crystallites of 3, thus stabilizing the gel systems and preventing further aggregation and precipitation of bigger crystallites. This assumption is also supported by the fact that if a hydrogel sample of $\mathbf{3 / 3 N a}$ (aqueous $\mathrm{NaOH}$ solution; initial $\mathrm{pH}=11.7$ ) is covered with a layer of desalted water and stored at room temperature for $24 \mathrm{~h}$, the macroscopic appearance of the gel changes. The gel surface gets rougher and needle-like crystallites remain. In order to elucidate the exact molecular arrangement of $\mathbf{3}$ in the hydrogel state, detailed X-ray- and neutron scattering experiments of the hydrogel samples are planned.

For morphological studies of the supramolecular aggregates of $\mathbf{3}$ / $3 \mathrm{Na}$ in aqueous $\mathrm{NaOH}$ solutions, scanning electron microscopy (SEM) studies of dried hydrogel samples and cryogenic transmission electron microscopy (cryo-TEM) experiments of solutions at different $\mathrm{pH}$ values were carried out. The hydrogel at $\mathrm{pH} 11.9$ features a network of ribbon-like fibers of several $\mu \mathrm{m}$ length and several hundreds of nm width (Fig. 3). Increasing the $\mathrm{pH}$ to 12.7 yields a solution of spherical micelles with a diameter of approx. $3.6 \mathrm{~nm}$ (see ESI, Fig. S3†). This is in good agreement with twice the molecular length of the amphiphile. Further increase of $\mathrm{pH}$ to 14.0 results in the formation of micellar superstructures reminiscent of stacks of disclike micellar aggregates (see ESI, Fig. S4 $\dagger$ ). The aggregation of $\mathbf{3 N a}$ into micellar superstructures in the case of the sample obtained from $\mathrm{pH} 14.0$ can be explained by two facts: The high $\mathrm{NaOH}$ content of the solution leads to a Hofmeister-type salting-out effect by increasing the non-polar intermolecular interactions of the amphiphiles. Furthermore, the presence of sodium ions can lead to a "bridging" effect of sodium carboxylate moieties thus resulting in an agglomeration of the sodium carboxylate-covered micelles. ${ }^{22}$ The tendency for the formation of "low curvature objects" such as ribbons (Fig. 3) and discs (ESI, Fig. S4†) can be explained by anisotropic arrangement of the amphiphiles guided by intermolecular hydrogen bonding.

The thermal stability of the hydrogels was investigated by microdifferential scanning calorimetry ( $\mu$-DSC). A hydrogel sample

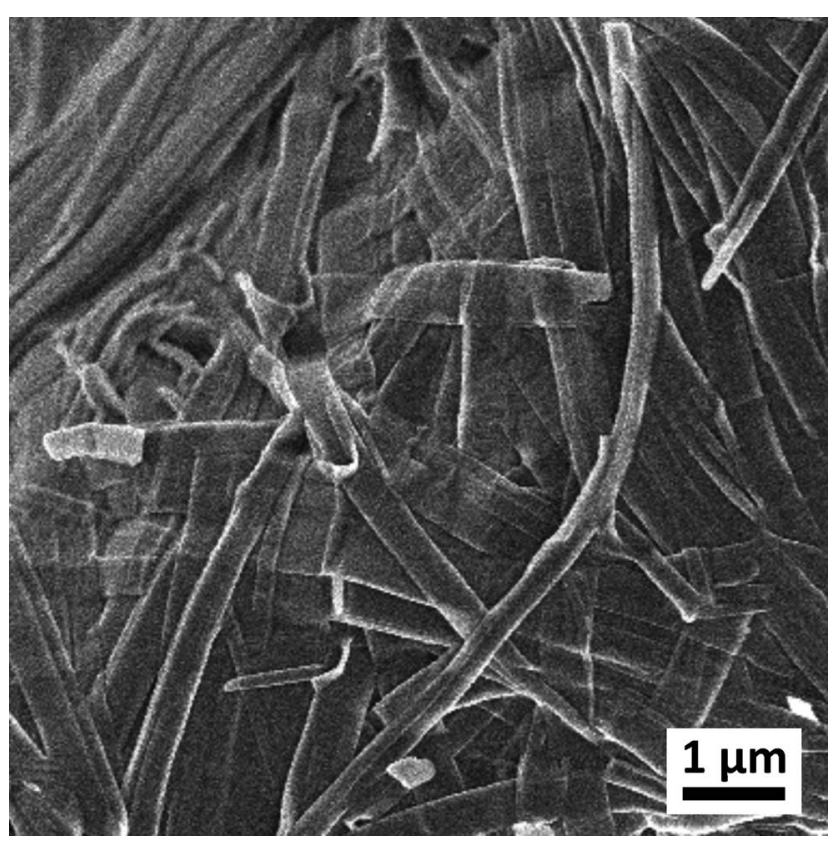

Fig. 3 SEM micrograph of a dried supramolecular hydrogel of $3 / 3 \mathrm{Na}$ exhibiting a collapsed network of ribbon-like aggregates (initial concentration $10 \mathrm{~g} \mathrm{~L}^{-1}$ of $\mathbf{3}$ in aqueous $\mathrm{NaOH}$ solution with an initial $\mathrm{pH}$ value of 11.9). 
prepared from $10 \mathrm{~g} \mathrm{~L}^{-1}$ of $\mathbf{3}$ in aqueous $\mathrm{NaOH}$ solution (initial $\mathrm{pH}=$ 11.9) was submitted to several subsequent tempering/heating/cooling cycles (see ESI, Fig. S5 and S6†). Upon heating, an endothermic peak is visible that can be attributed to the melting of the supramolecular assemblies of $\mathbf{3} / \mathbf{3 N a}$. Upon cooling/tempering, an exothermic peak (gel formation) can be recognized. It has to be noted that with increasing run number, a decrease in the melting enthalpy takes place (run \#1: $47.8 \mathrm{~J} \mathrm{~g}^{-1}$, run \#6: $41.9 \mathrm{~J} \mathrm{~g}^{-1}$, see ESI, Table $\mathrm{S} 4 \dagger$ ). Thus it is reasonable to assume that partial decomposition of the sample at elevated temperatures above $T_{\text {gel }}$ takes place. Due to the aqueous conditions, a hydrolysis of the amide bond can take place. This implies a thermally induced release of 4-hexyloxyaniline. In order to prove this assumption, HPLC experiments using a hydrogel sample (10 $\mathrm{g} \mathrm{L}^{-1}$ of 3 in aqueous $\mathrm{NaOH}$ solution, initial $\mathrm{pH} 11.9$ ) that was tempered at $85^{\circ} \mathrm{C}$ were carried out. The results clearly show that the initial drug molecule 4-hexyloxyaniline can be released thermally from the hydrogel of $\mathbf{3}$ (see ESI, Fig. S7 $\dagger$ ). Furthermore, we assume that an enzymatic cleavage of the amide bond and therefore enzymetriggered release of the initial drug is also possible. Finally, it is imaginable that the observed antibiotic and antimycobacterial properties of the amides 1-6 are based on the slow enzymatic hydrolysis of the amide bonds in vivo, thus releasing the underlying and bioactive 4-alkoxyanilines. Detailed investigation of this very promising class of compounds and studies on enzyme-triggered drug release are planned for the future.

\section{Conclusions}

We have demonstrated that it is possible to synthetically modify antimycobacterial 4-alkoxyanilines by amidation with diglycolic acid anhydride yielding amphiphilic low molecular weight hydrogelators. The resulting diglycolic acid monoamides retain specific and chainlength dependent antimicrobial and antimycobacterial activity while showing practically no antiproliferative and only weak to negligible cytotoxic effects. Especially the hexyloxy derivative $\mathbf{3}$ is able to reproducibly form thermoreversible hydrogels at concentrations down to $5 \mathrm{~g} \mathrm{~L}^{-1}$. The corresponding hydrogels feature ribbon-like network morphologies of the underlying supramolecular aggregates. The experimental results show that the initial drug molecule can be released from the hydrogel via hydrolysis.

\section{Acknowledgements}

We would like to thank the Hans-Knöll-Institute Jena for the evaluation of the biological activity, Martina Heider (BIMF/BZKG, University of Bayreuth) for the SEM investigations, Dr Markus Drechsler (BIMF/BZKG, University of Bayreuth) for conducting the cryo-TEM experiments, Birgit Brunner (Department of chemical engineering, Prof. Andreas Jess, University of Bayreuth) for performing the elemental analysis, Tobias Güttler for the synthesis and characterization of the amphiphiles and Doris Hanft (Macromolecular Chemistry I, University of Bayreuth) for conducting the HPLC experiments. Financial support from the German Research Foundation in the frame of SPP 1259 "Intelligente Hydrogele" is gratefully acknowledged.

\section{Notes and references}

1 A. Koul, E. Arnoult, N. Lounis, J. Guillemont and K. Andries, Nature, 2011, 469, 483; S. Ahmad, Respir. Res., 2010, 11, 169.

2 L. A. Estroff and A. D. Hamilton, Chem. Rev., 2004, 104, 1201; M. de Loos, B. L. Feringa and J. H. van Esch, Eur. J. Org. Chem., 2005, 3615.

3 K. Rajagopal, M. S. Lamm, L. A. Haines-Butterick, D. J. Pochan and J. P. Schneider, Biomacromolecules, 2009, 10, 2619.

4 Z. Yang, K. Xu, Z. Guo, Z. Guo and B. Xu, Adv. Mater., 2007, 19, 3152; Z. Yang, G. Liang, Z. Guo, Z. Guo and B. Xu, Angew. Chem., Int. Ed., 2007, 46, 8216.

5 G. Cheng, V. Castelletto, R. R. Jones, C. J. Connon and I. W. Hamley, Soft Matter, 2011, 7, 1326.

6 R. Orbach, L. Adler-Abramovich, S. Zigerson, I. Mironi-Harpaz, D. Seliktar and E. Gazit, Biomacromolecules, 2009, 10, 2646.

7 X.-D. Xu, L. Liang, C.-S. Chen, B. Lu, N.-L. Wang, F.-G. Jiang, X.-Z. Zhang and R.-X. Zhuo, ACS Appl. Mater. Interfaces, 2010, 2, 2663.

8 Z. Yang, K. Xu, L. Wang, H. Gu, H. Wei, M. Zhang and B. Xu, Chem. Commun., 2005, 4414.

9 J. Naskar, G. Palui and A. Banerjee, J. Phys. Chem. B, 2009, 113, 11787.

10 S. Cao, X. Fu, N. Wang, H. Wang and Y. Yang, Int. J. Pharm., 2008, $357,95$.

11 R. N. Mitra, D. Das, S. Roy and P. K. Das, J. Phys. Chem. B, 2007, 111, 14107.

12 A. Friggeria, B. L. Feringa and J. van Esch, J. Controlled Release, 2004, 97, 241.

13 A. Shome, S. Debnath and P. K. Das, Langmuir, 2008, 24, 4280.

14 S. Debnath, A. Shome, D. Das and P. K. Das, J. Phys. Chem. B, 2010, 114, 4407.

15 Z. Yang, H. Gu, Y. Zhang, L. Wang and B. Xu, Chem. Commun., 2004, 208

16 S. Bhuniya, Y. J. Seo and B. H. Kim, Tetrahedron Lett., 2006, 47, 7153.

17 Y. Gao, Y. Kuang, Z.-F. Guo, Z. Guo, I. J. Krauss and B. Xu, J. Am. Chem. Soc., 2009, 131, 13576.

18 P. K. Vemula, G. A. Cruikshank, J. M. Karp and G. John, Biomaterials, 2009, 30, 383.

19 Z. Yang and B. Xu, J. Mater. Chem., 2007, 17, 2358; J. C. Tiller, Angew. Chem., Int. Ed., 2003, 42, 3072.

20 E. Sim, M. Payton and J. Sinclair, WO 9961625 A1.

21 C. Tang, A. M. Smith, R. F. Collins, R. V. Ulijn and A. Saiani, Langmuir, 2009, 25, 9447.

22 K. Rosenlehner, B. Schade, C. Böttcher, C. M. Jäger, T. Clark, F. W. Heinemann and A. Hirsch, Chem.-Eur. J., 2010, 16, 9544; C. M. Jäger, A. Hirsch, B. Schade, K. Ludwig, C. Böttcher and T. Clark, Langmuir, 2010, 26, 10460; A. Bernet, M. Behr and H.-W. Schmidt, Soft Matter, 2011, 7, 1058. 